\title{
Homogeneous Graft Copolymerization of Vinyl Monomers onto Cellulose in a Dimethyl Sulfoxide-Paraformaldehyde Solvent System III. Methyl Acrylate
}

\author{
Noboru NishiOKA, Kouji MiNAMI, and Kouichi KOSAI \\ Faculty of Engineering, Osaka Electro-Communication University, \\ Neyagawa, Osaka 572, Japan
}

(Received March 16, 1983)

\begin{abstract}
Homogeneous graft copolymerization of methyl acrylate (MA) onto cellulose was carried out in a dimethyl sulfoxide-paraformaldehyde solvent system. Ammonium persulfate (APS), benzoyl peroxide (BPO), and azobisisobutyronitrile (AIBN) were used as radical initiators. The optimum grafting condition and the number of grafts per cellulose chain for each monomerinitiator system were determined as functions of polymerization temperature and concentrations of monomer, initiator, and cellulose. Grafting onto cellulose proceeded in the MA-APS system, but hardly at all in the MA-BPO and MA-AIBN systems. The number of grafts was found to be 1.5 at most in the MA-APS system. BPO was found unsuitable as a grafting initiator due to the degradation of the cellulose chains.

KEY WORDS Homogeneous Grafting / Cellulose / Methyl Acrylate / Dimethyl Sulfoxide-Paraformaldehyde Solvent System / Grafting Percentage / Grafting Efficiency / Number of Grafts /
\end{abstract}

Graft copolymerization onto cellulose is an effective method for modifying its properties. ${ }^{1-4}$ The characterization of the graft copolymers obtained would be necessary to elucidate their properties and the mechanism of graft copolymerization, since their physical and chemical properties depend on such parameters as the composition of the copolymer, the molecular weight of the backbone cellulose, the number of grafts per cellulose chain, and their molecular weight, etc.

It is generally accepted that the graft copolymerization of vinyl monomers onto cellulose in a heterogeneous system proceeds only in amorphous regions, causing the number of grafts seldom to exceed unity. ${ }^{5-9}$ However, it has been report$\mathrm{ed}^{10-12}$ that the number of grafts is more than unity in homogeneous graft copolymerization. A plausible reason for this may be that, in homogeneous systems, all the cellulose chains participate in the copolymerization.

The present study deals with the homogeneous graft copolymerization of methyl acrylate (MA) onto cellulose with radical initiators in a dimethyl sulfoxide (DMSO)-paraformaldehyde (PF) solvent system. The optimum conditions under which grafting onto cellulose occurs most effectively were determined. The characterization of the graft copolymers obtained under various grafting conditions was carried out.

\section{EXPERIMENTAL}

Bleached softwood sulfite pulp supplied by Fujibo Co. was used as the cellulosic material. Practical grade PF dried in a desiccator over $\mathrm{CaCl}_{2}$ and reagent grade DMSO were used without further purification. MA was purified by a standard procedure.

Thoroughly dried softwood pulp was dissolved in DMSO containing PF by a method described in our previous paper. ${ }^{10}$ The clear solution obtained, designated as the DMSO solution, was used as the stock cellulose solution. Adequate amounts of this solution and an initiator were mixed in a threenecked flask and purified nitrogen was bubbled through the solution for about $30 \mathrm{~min}$ at a temperature a little below $20^{\circ} \mathrm{C}$. The required amount of MA was then added. Polymerization was carried 
out at different fixed temperatures between 20 and $80^{\circ} \mathrm{C}$ and finally terminated by adding hydroquinone. The polymerization mixture was poured into a large amount of distilled water with vigorous stirring to precipitate the reaction product. The crude graft product was treated with acetone in a Soxhlet apparatus for more than $24 \mathrm{~h}$ to extract an attendant homopolymer, designated as extracted poly(methyl acrylate) (PMA). The infrared spectrum of the graft copolymer showed characteristic peaks of PMA, indicating that PMA was grafted onto the cellulose backbone.

The graft product was characterized by the following parameters:

$$
\begin{aligned}
& \text { weight conversion, WC (\%) } \\
& =\frac{\text { total weight of PMA formed }}{\text { weight of MA charged }} \times 100 \\
& \text { grafting percentage, GP }(\%) \\
& =\frac{\text { weight of graft PMA }}{\text { weight of cellulose }} \times 100 \\
& \text { grafting efficiency, GE (\%) } \\
& =\frac{\text { weight of graft PMA }}{\text { total weight of PMA formed }} \times 100 \\
& \text { number of grafts per cellulose chain } \\
& =\frac{\text { molecular weight of cellulose }}{\text { molecular weight of graft PMA }} \times \frac{\text { GP }}{100}
\end{aligned}
$$

Some of the graft products subjected to extraction were analyzed by thin layer chromatography (TLC) in order to detect any remaining homopolymer. A stock solution was prepared by dissolving a given sample in the DMSO-PF solvent according to the method described previously ${ }^{10}$ and an aliquot was applied on a TLC plate, Yamato Replate (Yamato Scientific Co.). The mixture of acetone and chloroform (2:1 by weight) was used as a developer. After development, chromatograms were stained with iodine vapor.

The intrinsic viscosity, $[\eta]$ (in $\mathrm{cm}^{3} \mathrm{~g}^{-1}$ ), of extracted PMA in acetone at $25^{\circ} \mathrm{C}$ was determined by an Ubbelohde type capillary viscometer. The following equation was used to estimate the viscosityaverage molecular weight. ${ }^{13}$

$$
[\eta]_{\text {acetone }}^{25^{\circ} \mathrm{C}}=5.5 \times 10^{-3} \mathrm{M}^{0.77}
$$

The molecular weight of the backbone cellulose was estimated to be $16 \times 10^{4}$ from $[\eta]$ obtained with the
DMSO-PF solvent at $30^{\circ} \mathrm{C}^{14}$ and with the cadoxen solvent at $20^{\circ} \mathrm{C} .{ }^{15}$

The viscosity measurement of the DMSO solution was made on a rotary viscometer, Vismetron VA-1 (Toshiba System Co.).

\section{RESULTS AND DISCUSSION}

Two separation problems must be elucidated for characterizing a graft copolymer. One is the separation of the attendant homopolymer from the crude graft product, which is associated with the estimation of the grafting percentage. The other is the separation of the graft polymer from the graft copolymer, which is associated with the estimation of the molecular weight of the graft polymer.

The difficulty in separating the attendant homopolymers from the crude graft products has been pointed out by many workers. ${ }^{3,6,16,17}$ Recently TLC was found effective for this separation. ${ }^{7,12,18,19}$ In this experiment, the crude graft product was subjected to extraction, followed by degradation of the cellulose backbone by acid hydrolysis. TLC was then used to separate the residue obtained following hydrolysis into two components, i.e., the attendant homopolymer and the true graft polymer having certain sugar residues at one of each polymer chain end. Thus, the analysis of the chromatograms made it possible to estimate the true grafting percentage. Quantitative analysis of chromatograms, however, was very tedious. In this work, therefore, not the residue obtained after hydrolysis, but the samples subjected to extraction were analyzed only qualitatively by TLC. The samples which showed no spots associated with PMA are discussed below.

It is well known that the backbone cellulose is so easily degraded by hydrolysis to low molecular weight residues that graft polymers can be isolated. ${ }^{2,3}$ The isolation of graft PMA was carried out with various concentrations of aqueous $\mathrm{NaOH}^{20}$ or $\mathrm{H}_{2} \mathrm{SO}_{4}{ }^{21,22}$ The isolated polymer, however, was not soluble in acetone and its infrared spectrum showed no peaks characteristic of PMA, indicating that the graft polymer was also hydrolyzed.

It is generally accepted that, in homogeneous grafting, the molecular weight of the graft polymer is similar to that of the attendant homopolymer. ${ }^{1-3}$ Moreover, both molecular weights were consistent with each other within experimental error in the homogeneous grafting of acrylonitrile (AN) and 
methyl methacrylate (MMA) onto cellulose in the DMSO-PF solvent system. ${ }^{11}$ In this work, therefore, the molecular weight of extracted PMA was used in place of that of graft PMA.

\section{Initiation with Peroxides}

Two peroxide initiators were tested for the grafting of MA onto cellulose. The results obtained with ammonium persulfate (APS) are discussed first.

Figure 1 shows the effect of polymerization temperature. As can be seen from Figure 1-a, the weight conversion and grafting percentage show maxima around $40^{\circ} \mathrm{C}$, indicating that an optimum temperature is present. The grafting efficiency decreases gradually with increasing temperature, implying that the activation energy of the propagation of PMA is larger than that of the formation of cellulose macroradicals. ${ }^{3}$ A similar temperature dependence of grafting efficiency has been found for other grafting systems. ${ }^{23}$

Figure 1-b shows the temperature dependence of the molecular weight of extracted PMA and the number of grafts per cellulose chain. The molecular weight of extracted PMA decreases with increasing temperature, whereas the number of grafts increases gradually and levels off at higher temperatures. These tendencies are consistent with the expectation based on the kinetics of radical polymerization. ${ }^{3}$ The decrease in grafting percentage at higher temperatures can be explained by the decrease in the

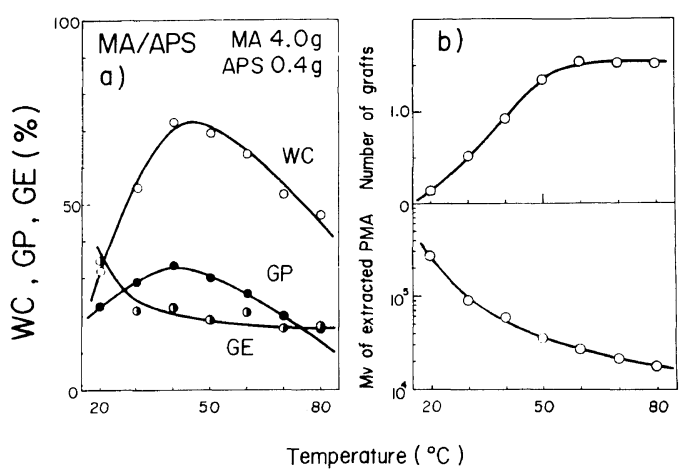

Figure 1. Effect of polymerization temperature on the grafting of MA with APS: (a) (O) weight conversion, WC; (○) grafting percentage, GP; (O) grafting efficiency, GE; (b) viscosity-average molecular weight, $M_{v}$, of extracted PMA and the number of grafts per cellulose chain; grafting time, $5 \mathrm{~h}$; cellulose concn, $2 \%$; MA concn, $4.0 \mathrm{~g} / 100 \mathrm{~g}$ DMSO soln; APS concn, $0.4 \mathrm{~g} / 100 \mathrm{~g}$ DMSO soln. molecular weight of graft PMA.

Figure 2 shows the effect of initiator concentration. The initiator concentration is expressed in grams of APS in $100 \mathrm{~g}$ of the DMSO solution and all experiments were carried out at $40^{\circ} \mathrm{C}$ which is, as seen from Figure 1, the optimum condition with respect to the weight conversion and grafting percentage. As the initiator concentration increases, the weight conversion increases sharply and levels off at about $80 \%$ and the grafting efficiency decreases gradually, whereas the grafting percentage shows a maximum around $0.4 \mathrm{~g} / 100 \mathrm{~g}$ and decreases gradually at higher APS concentrations.

The increase in initiator concentration probably causes both the increase in the number of grafts and the decrease in the molecular weight of the graft polymer. ${ }^{8,24-26}$ The results shown in Figure 2-b are consistent with this expectation. The decrease in grafting percentage at higher APS concentrations is attributed to the decrease in the molecular weight of
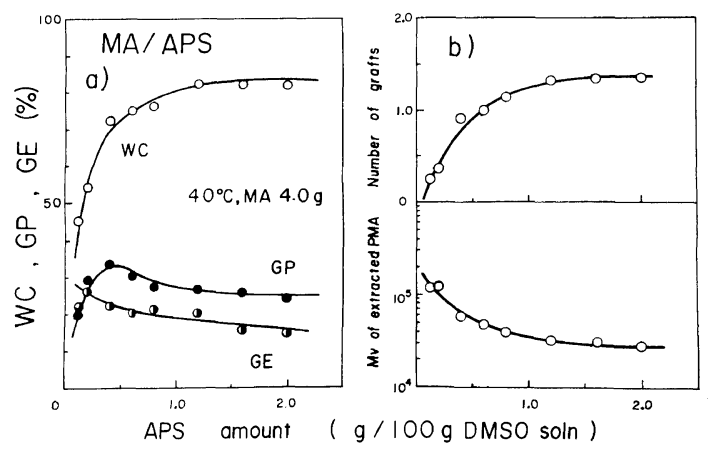

Figure 2. Effect of initiator concentration on the grafting of MA with APS: grafting time, $5 \mathrm{~h}$; cellulose concn, $2 \%$; polymerization temp, $40^{\circ} \mathrm{C}$.

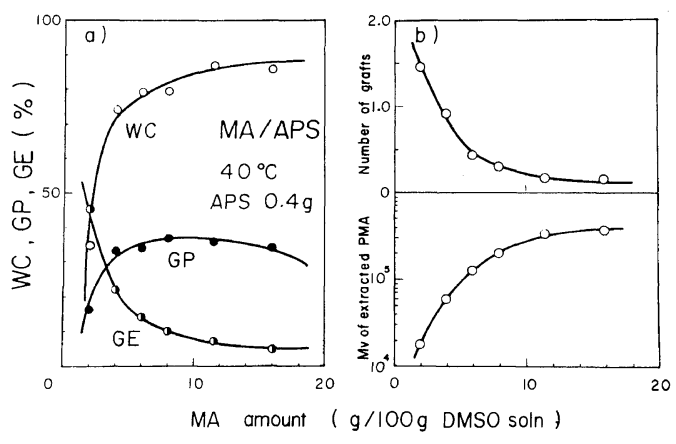

Figure 3. Effect of monomer concentration on the grafting of MA with APS: grafting time, $5 \mathrm{~h}$; cellulose concn, $2 \%$. 
graft PMA. The leveling off in the number of grafts at higher APS concentrations is ascribed to the saturation of the radical concentration, since a further increase in radical concentration would lead to the termination of primary radicals. This explanation may also account for the temperature dependence of the number of grafts shown in Figure 1b.

Figure 3 shows the effect of monomer concentration. The increase in monomer concentration is probably related to the increase in the molecular weight of the graft polymer. ${ }^{24-26}$ The monomer concentration is expressed in grams of MA in $100 \mathrm{~g}$ of the DMSO solution. The initiator concentration was kept at $0.4 \mathrm{~g} / 100 \mathrm{~g}$ which is, as seen from Figure 2 , the optimum condition with respect to the grafting percentage.

The increase in monomer concentration facilitates the consumption of primary radicals, thus reducing the formation of cellulose macroradicals and decreasing the grafting efficiency and the number of grafts. A similar monomer concentration dependence on grafting efficiency has been found for other grafting systems. ${ }^{27,28}$ The decrease in grafting percentage at higher monomer concentrations can be explained by the decrease in the number of grafts.

The broad maximum of the grafting percentage is comparable with our previous results obtained for the homogeneous grafting of AN and MMA onto cellulose in the DMSO-PF solvent system. ${ }^{10}$ In these graftings, the grafting percentage for each monomer system showed a sharp maximum at about $4 \mathrm{~g} / 100 \mathrm{~g}$. The addition of monomer which is a nonsolvent for cellulose to the DMSO solution resulted in gelation. The concentration of gelation decreased in the order of MA, MMA, and AN. This finding may explain the broad maximum of the grafting percentage shown in Figure 3-a. However, even in the case of $\mathrm{AN}$, the concentration was about $40 \mathrm{~g} / 100 \mathrm{~g}$ and the gelation did not take place at the concentration range where grafting was carried out. Furthermore, the intrinsic viscosities of cellulose measured in the DMSO-PFmonomer solvent systems hardly changed in the concentration range where the grafting percentage showed a sharp maximum. Thus, it seems reasonable to consider that the decrease in the number of grafts accompanying the increase in monomer concentration is due to the conjugated effect of the
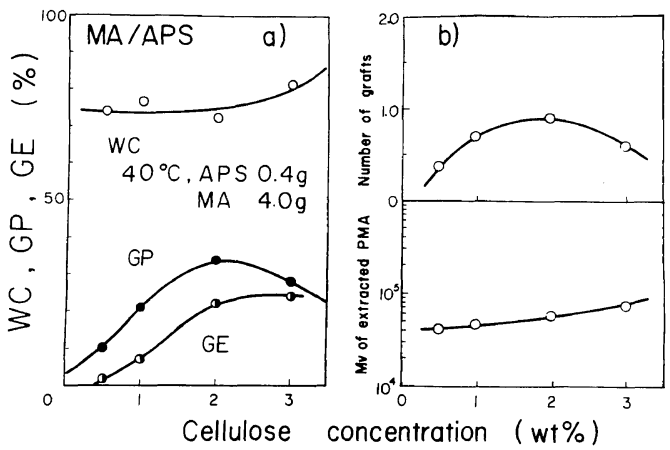

Figure 4. Effect of cellulose concentration on the grafting of MA with APS: grafting time, $5 \mathrm{~h}$.

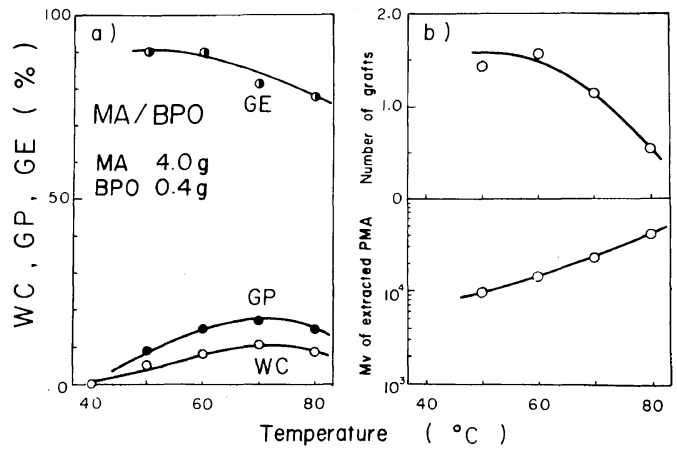

Figure 5. Effect of polymerization temperature on the grafting of MA with BPO: grafting time, $5 \mathrm{~h}$; cellulose concn, $2 \%$.

decrease in both the solubility of cellulose in the DMSO-PF-monomer solvent system and the primary radical concentration.

It is generally recognized that the grafting efficiency increases with increasing polymer concentration. ${ }^{3,24}$ The effect of cellulose concentration is shown in Figure 4. The DMSO solution of cellulose was so viscous that the concentration of cellulose could not be changed in a wide range. The weight conversion remains virtually constant over the concentration range examined, while the grafting percentage shows a maximum around $2 \%$ and the grafting efficiency increases gradually and then levels off. On the other hand, the molecular weight of extracted PMA increases slightly with increasing cellulose concentration and the number of grafts shows a maximum around $2 \%$. The increase in the molecular weight of extracted PMA and the decrease in the number of grafts at higher cellulose concentrations may be ascribed to the gel effect. The decrease in grafting percentage at higher cellulose 


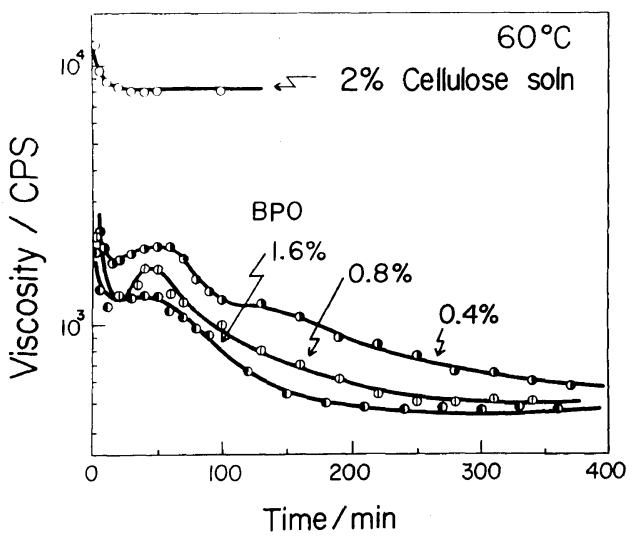

Figure 6. Effect of BPO concentration on the DMSO solution viscosity: cellulose concn, $2 \%$; temp, $60^{\circ} \mathrm{C} ;(\mathrm{O})$ DMSO soln containing no BPO; ( ) BPO concn, $0.4 \mathrm{~g} /$ $100 \mathrm{~g}$ DMSO soln; (1) BPO concn, $0.8 \mathrm{~g}$; (D) BPO concn, $1.6 \mathrm{~g}$.

concentrations can be explained by the decrease in the number of grafts.

Benzoyl peroxide (BPO) was also used as the initiator for the grafting of MA. Figure 5 shows the effect of polymerization temperature. The weight conversion and grafting percentage show maxima around $70^{\circ} \mathrm{C}$, whereas both of these values are $10 \%$ at most. On the other hand, the grafting efficiency is very high and decreases with increasing temperature. Furthermore, the molecular weight of extracted PMA increases with increasing temperature and the number of grafts decreases. These tendencies are not consistent with those shown in Figure 1. It was also found that, in this initiator system, the solution viscosity decreased sharply in the course of grafting. The decrease in solution viscosity was especially remarkable at higher temperatures and at higher initiator concentrations.

Figure 6 shows the effect of BPO concentration on the solution viscosity. The viscosity of the cellulose solution containing no BPO decreases with time and levels off. The decrease at the initial stage may be due to the time lag, until the temperature becomes constant. The viscosity of the DMSO solution containing BPO drops rapidly and then gradually. The higher the BPO concentration, the more sharply the viscosity decreases. This result indicates that the cellulose backbone was degraded by BPO and thus that BPO is not suitable as a grafting initiator. The solution viscosity also de-

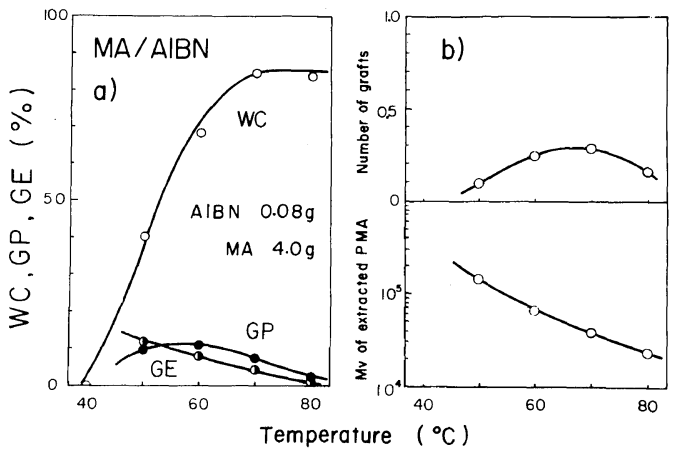

Figure 7. Effect of polymerization temperature on the grafting of MA with AIBN: grafting time, $5 \mathrm{~h}$; cellulose concn, $2 \%$.
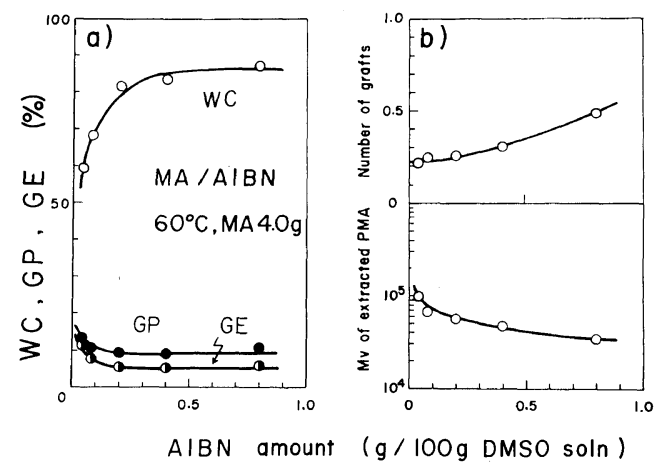

Figure 8. Effect of initiator concentration on the grafting of MA with AIBN: grafting time, $5 \mathrm{~h}$; cellulose concn, $2 \%$.

creased slightly in the case of other initiator systems such as the APS system discussed above and azobisisobutyronitrile (AIBN) system discussed below. However, degradation of the cellulose backbone in these initiator systems was neglected since the decrease in viscosity was slight over the initiator concentration range where grafting was carried out. The intrinsic viscosity measurement also indicated a similar result, ${ }^{29}$ i.e., the intrinsic viscosity of cellulose samples recovered from the DMSO solution containing an initiator decreased sharply with increasing initiator concentration in the BPO system but hardly changed in the APS and AIBN systems.

\section{Initiation with Azobisisobutyronitrile}

It is well known that AIBN is not suitable as a grafting initiator because of the resonance stabilization of its radical fragments. ${ }^{3}$ Homogeneous 
grafting onto cellulose in the DMSO-PF solvent system, however, indicated that AIBN was effective for the grafting of MMA but not for that of AN. ${ }^{10,11}$ Furthermore, the effectiveness of AIBN has been reported for the grafting of vinyl acetate onto cellulose in a heterogeneous system ${ }^{30}$ and that of vinyl monomers onto 6-nylon in a homogeneous system. ${ }^{31,32}$ Thus, the grafting of MA in the DMSO-PF solvent system was also carried out with AIBN as the initiator.

The effects of temperature and AIBN concentration are shown Figures 7 and 8, respectively. The weight conversion increases remarkably, while both the grafting percentage and grafting efficiency are $10 \%$ at most. Moreover, the number of grafts is 0.5 at most. Since the chain transfer from AIBN primary radicals to cellulose may be negligible due to resonance stabilization, these results show that the chain transfer from PMA homopolymer macroradicals to cellulose took place slightly in this monomer-initiator system, and that the chain transfer reactivity of PMA macroradicals to cellulose, as in the case of PAN macroradicals, was lower than that of PMMA macroradicals. ${ }^{10,11}$

\section{REFERENCES}

1. E. H. Immergut, "Encyclopedia of Polymer Science and Technology," Vol. 3, H. F. Mark, N. G. Gaylord, and N. B. Bikales, Ed., Interscience, New York, 1965, p 242.

2. V. T. Stannett and H. B. Hopfenberg, "Cellulose and Cellulose Derivatives," Vol. V, Part V, N. B. Bikales and L. Segal, Ed., Wiley, New York, 1971, p 907.

3. F. Ide, "Graft Jugo to Sono Oyo," Kobunshi Kankokai, Kyoto, 1977.

4. B. P. Morin, I. P. Breusova, and Z. A. Rogovin, $A d v$. Polym. Sci., 42, 139 (1982).

5. Y. Ogiwara, H. Kubota, and K. Arai, J. Appl. Polym. Sci., 14, 1049 (1970).

6. I. Sakurada, Y. Ikada, and Y. Nishizaki, J. Polym. Sci., C, No. 39, 265 (1972).

7. T. Taga and H. Inagaki, Angew. Makromol. Chem., 33, 129 (1973).

8. P. Lepoutre and S. H. Hui, J. Appl. Polym. Sci., 19, 1257 (1975).
9. A. Moze and S. Lapanje, Makromol. Chem., 180, 1599 (1979).

10. N. Nishioka and K. Kosai, Polym. J., 13, 1125 (1981).

11. N. Nishioka, K. Matsumoto, and K. Kosai, Polym. J., 15, 153 (1983).

12. M. Tsuzuki, I. Hagiwara, N. Shiraishi, and T. Yokota, J. Appl. Polym. Sci., 25, 2909 (1980).

13. M. Kurata, Y. Tsunashima, M. Iwama, and K. Kamide, "Polymer Handbook," 2nd ed, J. Brandrup and E. H. Immergut, Ed., Wiley-Interscience, New York, N. Y., 1975, Chapter IV.

14. H. A. Swenson, J. Appl. Polym. Symp., 28, 945 (1976).

15. E. Treiber, "Polymer Handbook," 2nd ed, J. Brandrup and E. H. Immergut, Ed., WileyInterscience, New York, N. Y., 1975, Chapter V.

16. H. Yasuda, J. A. Wary, and V. T. Stannett, J. Polym. Sci., C, 2, 387 (1963).

17. H. A. Ende and V. T. Stannett, J. Polym. Sci., A, No. 2, 4047 (1964).

18. H. Inagaki, T. Kotaka, and T. I. Min, Pure Appl. Chem., 46, 61 (1973).

19. T. I. Min and H. Inagaki, Polymer, 21, 309 (1980).

20. P. Cremonest, Cellulose Chem. Technol., 2, 459 (1968).

21. F. Ide, Kogyo Kagaku Zasshi, 64, 1489 (1961).

22. R. Y. M. Huang, B. Immergut, E. M. Immergut, and W. H. Rapson, J. Polym. Sci., A, 1, 1257 (1963).

23. T. Maekawa, K. Hayashi, K. Uno, and S. Okamura, Kobunshi Kagaku, 21, 323 (1964).

24. Yo. Ogiwara, Yu. Ogiwara, and H. Kubota, J. Polym. Sci., A-1, 5, 2791 (1967).

25. V. Hornof, B. V. Kokta, and J. L. Valade, J. Appl. Polym. Sci., 19, 545 (1975).

26. V. I. Kurliankina, V. A. Molotkov, S. I. Klenin, and S. Ya. Liubina, J. Polym. Sci., Polym. Chem. Ed., 18, 3369 (1980).

27. Y. Minoura and Y. Mori, Kogyo Kagaku Zasshi, 61, 109 (1958).

28. F. Ide, K. Nakatsuka, and H. Tamura, Kobunshi Kagaku, 23, 45 (1966).

29. N. Nishioka and K. Monmae, in preparation.

30. B. M. Misra, J. K. Jassal, and C. P. Pande, J. Polym. Sci., Polym. Chem. Ed., 16, 295 (1978).

31. K. Kosai, T. Higashino, and S. Ohue, Nippon Kagaku Kaishi, 523 (1975).

32. K. Kosai, T. Higashino, and N. Nishioka, Nippon Kagaku Kaishi, 561 (1977). 